OPEN ACCESS

Edited by:

Zhaolai Chen

Shandong University, China

Reviewed by:

Andrew Nattestad,

University of Wollongong, Australia

Luca Tortora

Roma Tre University, Italy

*Correspondence:

Xin Wang

230159424@seu.edu.cn

Wei Lei

Iw@seu.edu.cn

Specialty section:

This article was submitted to

Inorganic Chemistry,

a section of the journal

Frontiers in Chemistry

Received: 25 May 2020

Accepted: 03 August 2020

Published: 15 September 2020

Citation:

Xu Y, Wang X, Pan Y, Li Y, Emeka Elemike E, Li Q, Zhang X, Chen J,

Zhao Z and Lei W (2020) Perovskite

Photodetectors Based on p-i-n

Junction With Epitaxial

Electron-Blocking Layers.

Front. Chem. 8:811

doi: $10.3389 /$ fchem.2020.00811

\section{Perovskite Photodetectors Based on p-i-n Junction With Epitaxial Electron-Blocking Layers}

\author{
Yubing Xu ${ }^{1}$, Xin Wang ${ }^{1 *}$, Yuzhu Pan ${ }^{1}$, Yuwei $L^{1}$, Elias Emeka Elemike ${ }^{2}$, Qing $L i^{1}$, \\ Xiaobing Zhang ${ }^{1}$, Jing Chen ${ }^{1}$, Zhiwei Zhao ${ }^{1}$ and Wei Lei ${ }^{1 *}$ \\ ${ }^{1}$ School of Electronic Science and Engineering, Joint International Research Laboratory of Information Display and \\ Visualization, Southeast University, Nanjing, China, ${ }^{2}$ Chemistry Department of North West University, Potchefstroom, \\ South Africa
}

Organic-inorganic hybrid perovskite single crystals (PSCs) have been emerged as remarkable materials for some optoelectronic applications such as solid-state photodetectors, solar cells and light emitting diodes due to their excellent optoelectronic properties. To decrease the dark current, function layers based on spin-coating method are frequently requested for intrinsic PSCs to block the injected current by forming energy barrier. However, the amorphous function layers suffer from small carrier mobility and high traps density, which limit the speed of the photoelectric response of perovskite devices. This work supposes to grow thick $\mathrm{MAPbBr}_{3}$ and $\mathrm{MAPbl}_{3}$ mono-crystalline thin films on the surface of intrinsic $\mathrm{MAPbBr}_{2.5} \mathrm{Cl}_{0.5}$ PSCs substrate by a heteroepitaxial growth technique to act as electron-blocking layers. Meanwhile, C60 and [6,6]-phenyl-C61-butyric acid methyl ester (PCBM) layers are deposited on the opposite surface of substrate PSCs by spin-coating method to block injected holes. This Au-MAPbl $3-\mathrm{MAPbBr}_{3}-\mathrm{MAPbBr}_{2.5} \mathrm{Cl}_{0.5} \mathrm{PSCs}-\mathrm{C} 60-\mathrm{PCBM}-\mathrm{Ag}$ heterostructure can be used as excellent $\mathrm{X}$-ray photodetector (XPD) due to its low dark current density of $6.97 \times 10^{-11} \mathrm{~A} \mathrm{~cm}^{-2}$ at $-0.5 \mathrm{~V}$ bias, high responsivity of $870 \mathrm{~mA} / \mathrm{W}$ at $-100 \mathrm{~V}$ bias and X-ray sensitivity as high as $59.7 \mu \mathrm{C} \mathrm{mGy}{ }^{-1} \mathrm{~cm}^{-2}$ at $-50 \mathrm{~V}$ bias.

Keywords: photodetectors, self-powered, perovskite single crystals, epitaxial growth, X-ray detection

\section{INTRODUCTION}

The last few years have witnessed the intensive investigation on Organic-inorganic hybrid perovskite single crystals (OIHPSCs) by many researchers due to their advantages of high photons absorption coefficients, high charges mobility and adjustable band gaps (Dou et al., 2014). Besides, self-powered photodetectors based on $\mathrm{p}-\mathrm{n}$ junction and heterostructure can work stably without the applied voltage, which results in their improvement. Moreover, solution-processed optoelectronic devices have shown lower cost compared to those fabricated by vacuum deposition (Dong et al., 2015a; Miao and Zhang, 2019). Especially, OIHPSCs could be grown from solution with bulk size up to centimeter-scale and have shown attractive potential in X-ray detection and gamma ray detection (Wei et al., 2016). In that case, self-powered photodetectors based on solution-processed OIHPSCs tend to have better performance.

The common chemical formulas of OIHP are $\mathrm{MAPbX}_{3}$ (where $\mathrm{MA}=\mathrm{CH}_{3} \mathrm{NH}_{3}, \mathrm{X}$ can be $\mathrm{Br}$, $\mathrm{Cl}$ or I). The previous studies have demonstrated that diverse solution-processed function layers 


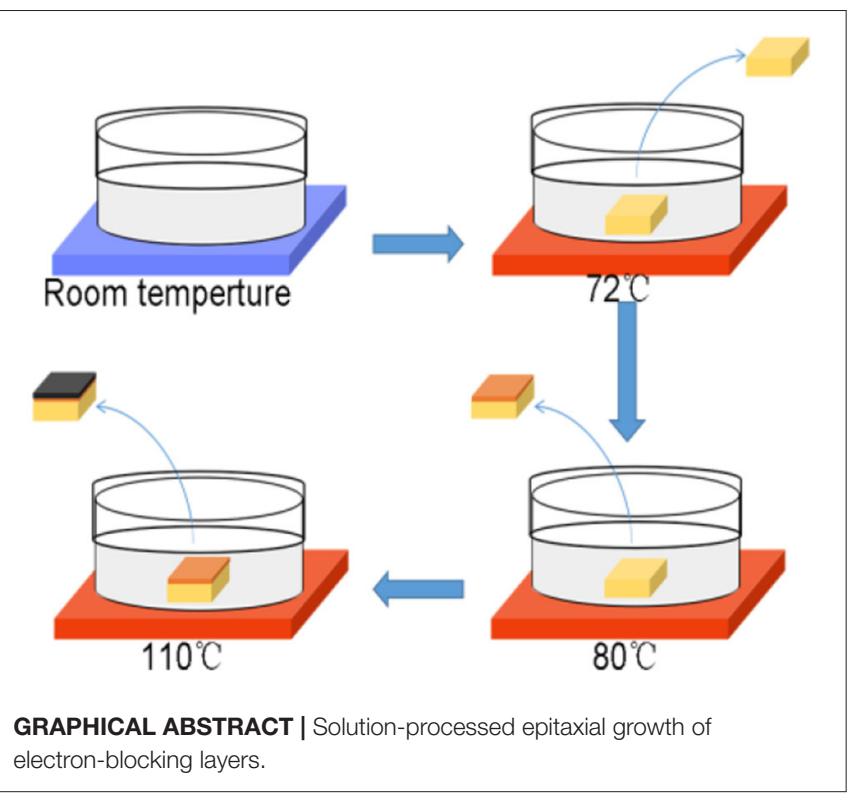

deposited on substrate crystals are generally used to fabricate photodetectors because of their transporting of photongenerated charges as well as their blocking of injected current under reverse bias (Wei et al., 2017; Xin et al., 2018; Jariwalad and Hersammc, 2017). To establish p-i-n junctions and decrease the dark current, the function layers based on solutionprocessed are requested for intrinsic $\mathrm{MAPbBr}_{2.5} \mathrm{Cl}_{0.5}$ PSCs. This sandwich structure consists of electron transporting layer (ETL), intrinsic PSCs and hole transporting layer (HTL). For example, N4,N40-bis(4-(6-((3-ethyloxetan-3-yl)methoxy)hexyl)-phenyl)N4,N40-diphenylbiphenyl-4,40-diamine (OTPD) and N,N0bis(3-methylphenyl)-N,N0-diphenylbenzidine (poly-TPD), which transport holes, are regarded as p-type semiconductors. And ETLs such as C60, [6,6]-phenyl-C61-butyric acid methyl ester (PCBM) and 2,9-dimethyl-4,7-diphenyl-1,10phenanthroline (BCP), which transport electrons, are regarded as n-type semiconductors (Wang and Kim, 2017; Wang et al., 2018). In principle, the conduction band energy of intrinsic PSCs should be lower than n-type layer, so that electrons will move to the conduction band of the function layers. And holes will move to the valence band of the inorganic function layers because PSCs' valence band energy should be higher than p-type layer (Sutherland and Sargent, 2016).

However, function layers deposited by spin-coating technique have nanoscale thickness and are easily generating defects, which lead to high traps density and large leakage current (Dong et al., 2015b; Kim et al., 2017). In addition, the function layers have low mobility resulting in difficult transportation of charges. What's more, lattice mismatching is unavoidable between function layers and PSCs substrate. As a result, the devices made by spincoating method suffer from large inner capacitance due to the accumulation of charges near interface region, which limits the speed of photoelectric response (Xin et al., 2018; Zhao et al., 2014). This work fabricates the photodetector with spin-coated
PCBM/C60 films on $\mathrm{MAPbBr}_{2.5} \mathrm{Cl}_{0.5}$ PSCs for comparison, the dark current density is $4.24 \times 10^{-8} \mathrm{~A} \mathrm{~cm}^{-2}$ at low bias voltage, which is not suitable for weak signal detection. Additionally, the weak tolerance to radiation of $\mathrm{MAPbBr}_{3}$ PSC with spincoated function layers results in added noise for high-energy ray detection has been reported (Xin et al., 2018). We fabrictes the compared device with spin-coated PCBM/C60 films on $\mathrm{MAPbBr}_{2.5} \mathrm{Cl}_{0.5}$ PSCs Recently, through changing the halide ratio, such as $\mathrm{MAPbI}_{3-\mathrm{x}} \mathrm{Br}_{\mathrm{x}}$ and $\mathrm{MAPbBr}_{3-\mathrm{x}} \mathrm{Cl}_{\mathrm{x}}$, the band gap of OIHPSCs can be modulated from 1.5 to $2.9 \mathrm{eV}$ ( $\mathrm{Hu}$ et al., 2016; Fang et al., 2015; Jiang et al., 2019). Among these OIHPSCs, $\operatorname{MAPbBr}_{(\mathrm{x}<2.5)} \mathrm{Cl}_{(3-\mathrm{x})}$ PSCs, OIHP single crystals become more $\mathrm{n}$-type with $\mathrm{x}$ decreasing. On the other hand, $\operatorname{MAPbBr}_{(\mathrm{x}>2.5)} \mathrm{Cl}_{(3-\mathrm{x})}$ and $\mathrm{MAPbBr}_{(3-\mathrm{x})} \mathrm{I}_{\mathrm{x}}$ PSCs become more $\mathrm{p}-$ type with $\mathrm{x}$ increasing as shown in Figure 1A (Wang et al., 2014; Jiang et al., 2019).

Therefore, we suppose to employ heteroepitaxial growth technique by solution-processed to form $\mathrm{p}$-i-n junctions. According to the lattice match rule, high quality perovskite layers have to be grown directly on the surface of substrate OIHPSCs in its precursor solution during crystallization process. In this work, we demonstrate that epitaxial perovskite films of high crystallinity could be grown on OIHPSCs. Compared with the spin-coated function layers, epitaxial perovskite films increase the carrier mobility and decrease the trap density. By controlling the time of epitaxial growth in precursor solution, the thickness of perovskite layers can be controlled in micrometer scale, which ensures the devices work stably under high voltage bias. In that case, our perovskite device of centimeter-scale thickness is mainly dedicated to high-energy ray detection such as $\mathrm{X}$-ray detection. Herein, we fabricate the XPD with $\mathrm{MAPbBr}_{2.5} \mathrm{Cl}_{0.5}$ PSCs as substrate crystals. Then epitaxial $\mathrm{MAPbBr}_{3}$ mono-crystalline thin films and $\mathrm{MAPbI}_{3}$ mono-crystalline thin films replace spincoated organic function layers as p-type, meanwhile PCBM and $\mathrm{C} 60$ films act as n-type. The epitaxial $\mathrm{MAPbBr}_{3}$ layers are used as buffer layers to satisfy the lattice matching condition for epitaxial $\mathrm{MAPbI}_{3}$ layers and $\mathrm{MAPbBr}_{2.5} \mathrm{Cl}_{0.5}$ PSCs. This XPD has shown low dark current density of $6.97 \times 10^{-11} \mathrm{~A} \mathrm{~cm}^{-2}$ at $-0.5 \mathrm{~V}$ bias, average electrons mobility of $385.9 \mathrm{~cm}^{2} \mathrm{~V}^{-1} \mathrm{~s}^{-1}$, high responsivity of $870 \mathrm{~mA} / \mathrm{W}$ at $-100 \mathrm{~V}$ bias, average noise current of $1.34 \mathrm{nA} \mathrm{Hz}^{-0.5}$ in darkness under $-100 \mathrm{~V}$ bias as well as high X-ray sensitivity of $59.7 \mu \mathrm{C} \mathrm{mGy}^{-1} \mathrm{~cm}^{-2}$ at -50 $\mathrm{V}$ bias.

\section{MATERIALS AND METHODS}

Materials: Lead iodide $\left(\mathrm{PbI}_{2}, 99 \%\right)$, lead bromine $\left(\mathrm{PbBr}_{2}, 98 \%\right)$ and Lead chloride $\left(\mathrm{PbCl}_{2}, 99 \%\right)$ were purchased from Sigma Aldrich. Methylamine alcohol solution (30-33 wt. \% in ethanol) and Hydroiodic Acid (40\%) and Hydrobromic Acid solution (55\%) were obtained from Macklin. PCBM and C60 were purchased from Aladdin. All commercial products were used as received.

Device fabrication: The precursor is a N,Ndimethylformamide (DMF) solution which dissolves $1 \mathrm{M}$ $\mathrm{MABr}, \mathrm{PbBr}_{2}$ and $\mathrm{PbCl}_{2}$ with a ratio of 1:0.75:0.25. After totally 
reacting and cooling down, the precursor solution were heated in crystallizing dish for $90 \mathrm{~h}$ from 50 to $75^{\circ} \mathrm{C}$ at a rate of $0.3^{\circ} \mathrm{C}$ h-1 for growth of high-quality $\mathrm{MAPbBr}_{2.5} \mathrm{Cl}_{0.5}$ PSCs. $1 \mathrm{M} \mathrm{MABr}$, $\mathrm{PbBr}_{2}$ with a ratio of $1: 1$ are dissolved in a DMF solution for epitaxial growth of MAPbBr3 mono-crystalline thin films at $80^{\circ} \mathrm{C}$. And $1 \mathrm{M}$ MAI, $\mathrm{PbI}_{2}$ with a ratio of $1: 1$ are dissolved in a $\gamma$-GBL solution to grow $\mathrm{MAPbI}_{3}$ mono-crystalline thin films at $110^{\circ} \mathrm{C}$. The programmable heating control system was realized on IKA-RET control-visc.

Spin-coating method is used to form C60 and PCBM function films respectively on the bottom surface of $\mathrm{MAPbBr}_{2.5} \mathrm{Cl}_{0.5}$ PSCs at 2500 r.p.m for 20s. C60, PCBM and sliver electrode solution were deposited on the opposite face by thermal evaporation in vacuum. $\mathrm{MAPbBr}_{3}$ mono-crystalline thin films, $\mathrm{MAPbI}_{3}$ monocrystalline thin films and gold electrode were deposited on opposite surfaces of PSCs.

Characterization: SEM images were taken with a Quanta 200 FEI (USA). XRD patterns were obtained by X'TRA (Switzerland). PL spectra were measured by UV-vis spectroscopy (Lab Tech Bluestar, USA).

Device Performance Measurements: Keithley 2410 was used as the voltage source to measure J-V curves. Noise of the device was characterized with a $450 \mathrm{~nm}$ LED at $10 \mathrm{~Hz}$ and Keithley 2410 as voltage source. The $460 \mathrm{~nm}$ light source is obtained from the F5 LED of GEESLED Company. A $355 \mathrm{~nm}$ pulsed Nd:YAG laser with $6 \mathrm{~ns}$ pulse width at $20 \mathrm{~Hz}$ was used as the illumination source and response time was measured using an Agilent oscilloscope with a Keithley 2410 as the voltage source. The X-ray source is obtained from PERLOVE Medical Company and the X-ray dose rate was obtained by a commercial dosimeter (FJ-347A, China).

\section{RESULTS AND DISCUSSION}

PSCs used in this work are grown by variable temperature crystallization (VTC) method which has been previously reported. (Stoumpos et al., 2013; Maculan et al., 2015) The fabrication procedure of $\mathrm{MAPbBr}_{2.5} \mathrm{Cl}_{0.5}$ PSCs and epitaxial perovskite mono-crystalline films is depicted in Figure 1B. As illustrated in Figure 1C, the size of $\mathrm{MAPbBr}_{2.5} \mathrm{Cl}_{0.5} \mathrm{PSC}$ is approximately $18 \mathrm{~mm} \times 18 \mathrm{~mm}$ in width and length, as well as $5 \mathrm{~mm}$ in thickness. The $\mathrm{MAPbBr}_{2.5} \mathrm{Cl}_{0.5}$ PSC is dipped into $\mathrm{MAPbBr}_{3}$ and $\mathrm{MAPbI}_{3}$ perovskite precursor solution respectively at optimum growth temperature. Bragg equation is used to calculate the lattice constants of $\mathrm{MAPbBr}_{2.5} \mathrm{Cl}_{0.5}$, $\mathrm{MAPbBr}_{3}$ and $\mathrm{MAPbI}_{3}$ (Jiang et al., 2019; Ji et al., 2018). Additionally, X-ray diffraction patterns of each single crystal are shown in Figure $\mathbf{S 1}$ for comparison. And the lattice constants of $\mathrm{MAPbBr}_{2.5} \mathrm{Cl}_{0.5}, \mathrm{MAPbBr}_{3}$ and $\mathrm{MAPbI}_{3}$ are $5.87 \AA$, 5.93 $\AA$ and $6.23 \AA$, respectively. Besides, the lattice mismatching rates of $\mathrm{MAPbBr}_{2.5} \mathrm{Cl}_{0.5}-\mathrm{MAPbBr}_{3}$ and $\mathrm{MAPbBr}_{3}-\mathrm{MAPbI}_{3}$ are calculated to be only 1.01 and $4.81 \%$. As a result, $\mathrm{MAPbBr}_{3}$ and $\mathrm{MAPbI}_{3}$ mono-crystalline thin films are formed on the surface of the $\mathrm{MAPbBr}_{2.5} \mathrm{Cl}_{0.5}$ PSCs in sequence. After cutting and polishing process, the epitaxial mono-crystalline thin films exist only on the top of substrate. As shown in Figure 1D, C60 and PCBM films, which act as n-type materials, are deposited on the bottom of $\mathrm{MAPbBr}_{2.5} \mathrm{Cl}_{0.5}$ PSCs via spin-coating method. The gold and silver electrodes are deposited on the top and bottom surfaces of the PSCs by thermal evaporation in vacuum. Figure 1E depicts the $\mathrm{Au}-$ $\mathrm{MAPbI}_{3}-\mathrm{MAPbBr}_{3}-\mathrm{MAPbBr}_{2.5} \mathrm{Cl}_{0.5}-\mathrm{C} 60-\mathrm{PCBM}-\mathrm{Ag}$ structure of

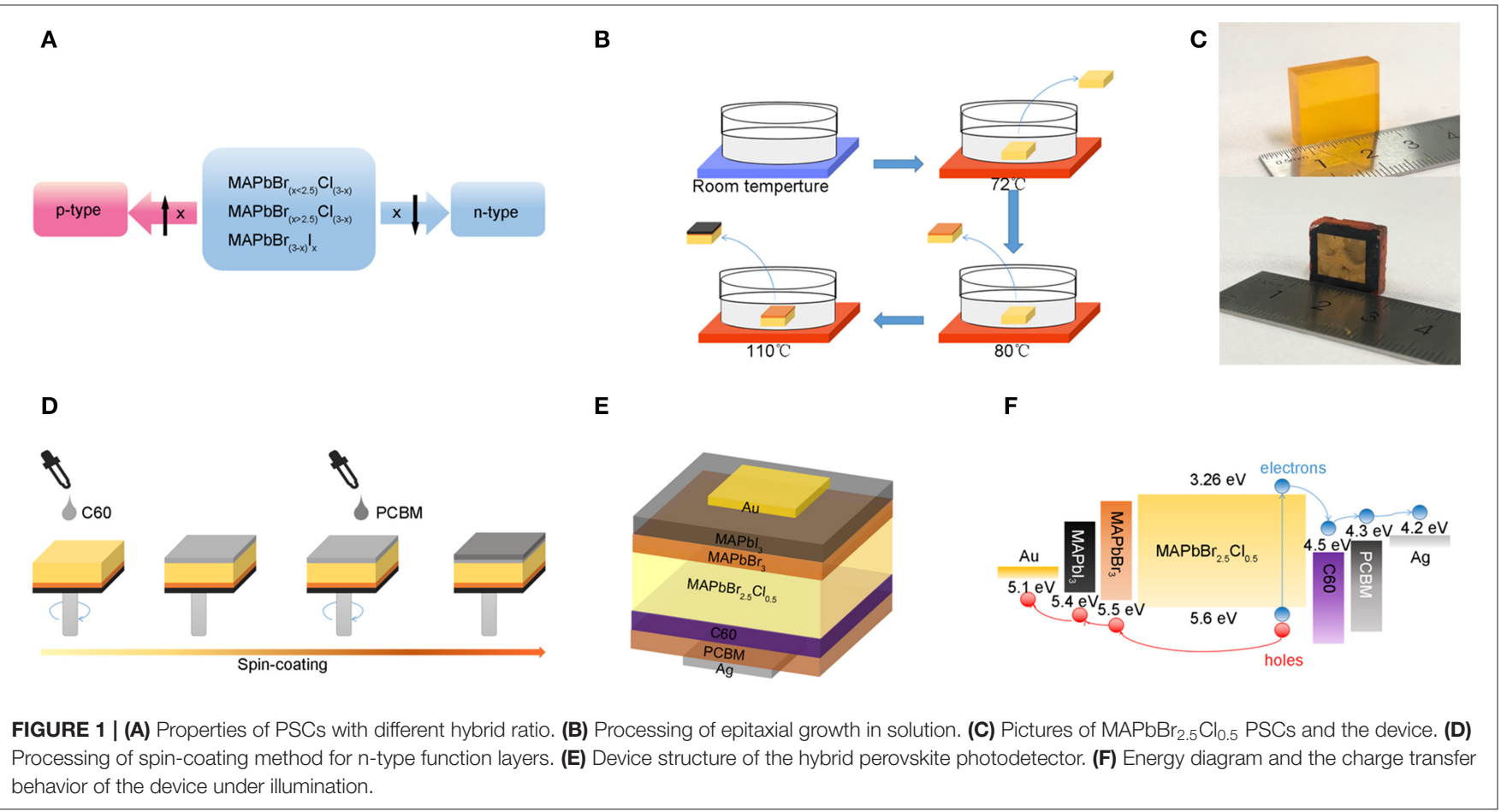



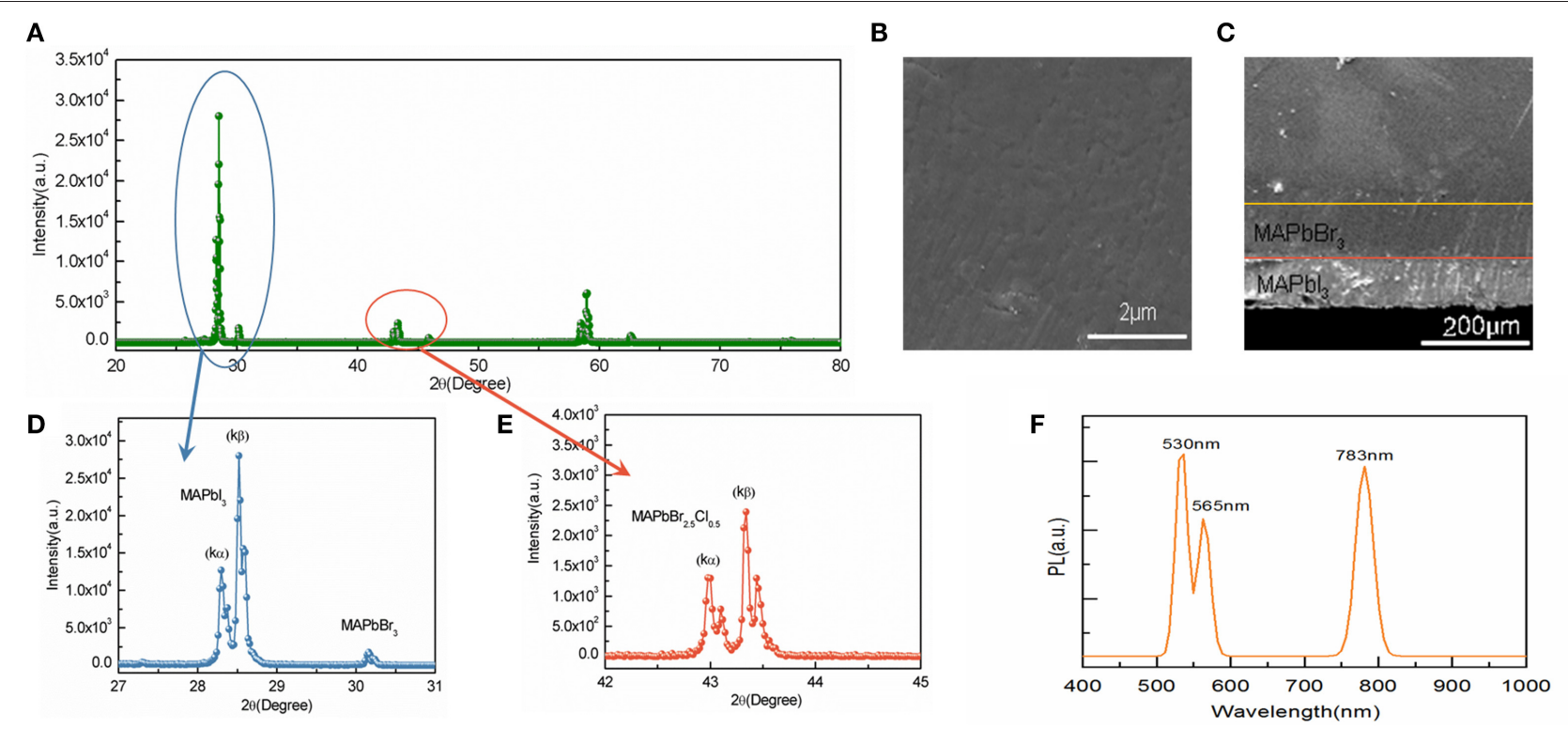

FIGURE 2 | (A) X-ray diffraction (XRD) pattern of epitaxial device. (B) Scanning electron microscope (SEM) image of the epitaxial MAPbl 3 film surface. (C) SEM image of the cross section of epitaxial films on substrate. (D) XRD pattern with Bragg angle from 27 to $31^{\circ}$. (E) XRD pattern with Bragg angle from 42 to $45^{\circ}$. (F) Photoluminescence (PL) spectra of the perovskite photodetector (with excitation wavelength of $450 \mathrm{~nm}$ ).

our photodetector. The energy diagram and the charge transfer behavior of this XPD with incident light from silver electrode are shown in Figure 1F. These photogenerated electrons and holes are transmitted by ETLs and HTLs and then are collected separately by the electrodes under external electric field. Moreover, C60 and PCBM films can block holes while $\mathrm{MAPbI}_{3}$ and $\mathrm{MAPbBr}_{3}$ mono-crystalline thin films block electrons injected by voltage source, which mainly benefit dark current (Bao et al., 2017).

As shown in Figure 2A, X-ray diffraction (XRD) is used to characterize the crystallinity of $\mathrm{MAPbBr}_{3}$ films and $\mathrm{MAPbI}_{3}$ films on the surface of $\mathrm{MAPbBr}_{2.5} \mathrm{Cl}_{0.5}$ PSCs. Scanning electron microscope (SEM) characterization of the surface of $\mathrm{MAPbI}_{3}$ mono-crystalline thin films layer on $\mathrm{MAPbBr}_{3}$ mono-crystalline thin films is shown in Figure 2B. Moreover, Figure 2C illustrates the cross-sectional view of the device in which the thickness of each epitaxial crystal layer is clearly visible, with approximately $110 \mu \mathrm{m}$ of $\mathrm{MAPbBr}_{3}$ film and $100 \mu \mathrm{m}$ of $\mathrm{MAPbI}_{3}$ film. Additionally, Energy Dispersive XRay (EDX) spectra of substrate and each layer are illustrated in Figure S1 to confirm the layers' thickness. Herein, it can be concluded by our experiments that epitaxial mono-crystalline thin films based on lattice matching have considerable thickness, excellent crystallinity (Pellegrino et al., 2015). Additionally, Figure 2D illustrates that the epitaxial films deposited on structure have a clear $\mathrm{MAPbI}_{3}$ peak with full width at half-maximum (FWHM) of $0.105^{\circ}$ and this strong peak is separated into $\mathrm{k} \alpha$ and $\mathrm{k} \beta$ peaks, which confirms high crystallization quality. In addition, there is a $\mathrm{MAPbBr}_{3}$ peak with FWHM of $0.062^{\circ}$. Similarly, as shown in Figure 2E, the FWHM of $\mathrm{MAPbBr}_{2.5} \mathrm{Cl}_{0.5}$ is $0.112^{\circ}$. For comparison, the XRD patterns of each single crystal are shown in Figure S2.

Figure 2F shows the Photoluminescence (PL) spectra (excited by $450 \mathrm{~nm}$ ) of $\mathrm{MAPbBr}_{2.5} \mathrm{Cl}_{0.5}$ PSC substrate with epitaxial growth of $\mathrm{MAPbBr}_{3}$ and $\mathrm{MAPbI}_{3}$ mono-crystalline thin films. Three emission peaks are obtained at wavelength of $530 \mathrm{~nm}$, $565 \mathrm{~nm}$ and $783 \mathrm{~nm}$ respectively with a LED excitation at $450 \mathrm{~nm}$. In addition, the PL spectra of each single crystal is provided in Figure S3. For comparison, the PL peaks of this heterostructure device obviously become more red-shifted than those of perovskite single crystals. According to previous literatures, the soft and dynamic crystal lattice of halide perovskites are attributed to the low energy barrier for perovskite crystal formation, which easily leads to solid-state ion migrations in the adjacent perovskite lattice (Shewmon et al., 2016; Pan et al., 2018; Zhang et al., 2019). During the process of epitaxial growth in this work, the $\mathrm{MAPbBr}_{2.5} \mathrm{Cl}_{0.5}-\mathrm{MAPbBr}_{3}$ and the $\mathrm{MAPbBr}_{3}-\mathrm{MAPbI}_{3}$ perovskite heterostructures are fabricated respectively where chloride-bromide and bromide-iodide interdiffusion across the perovskite heterojunction occur which leads to the red-shifted of PL peaks.

To characterize the electric properties of this photodetector for visible light, a white light emitting diode with adjustable illumination density is used to radiate gold electrode of the device. Due to the dark current denisity of photodetectors should be as low as possible in order to recognize weak signal current from noise (Nakamura et al., 2001; Li et al., 2020). The current density-voltage curves of our device under the sweep voltage from $-100 \mathrm{~V}$ to $100 \mathrm{~V}$ at different illumination denisty are shown in Figure 3A. Thanks to the epitaxial mono-crystalline films working as blocking layers, the dark current density of this device 

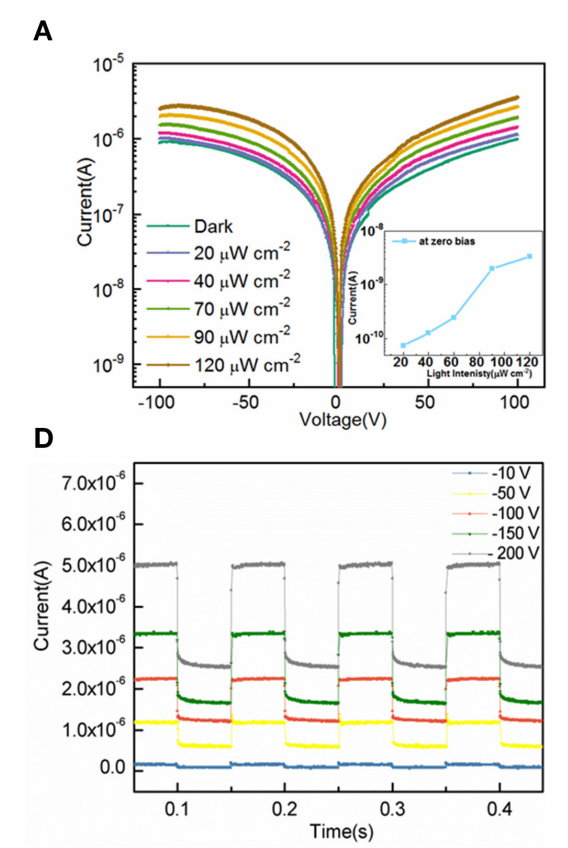

B

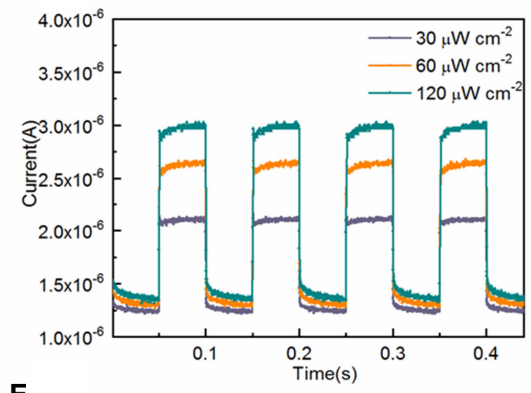

E

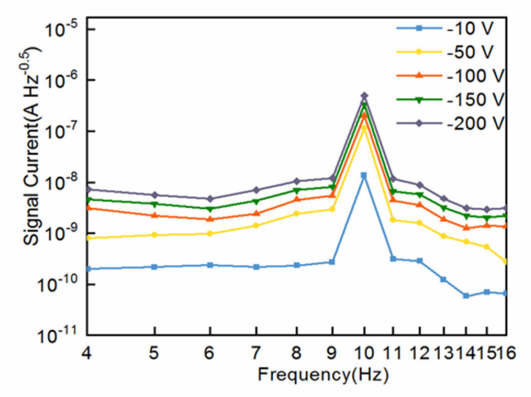

C

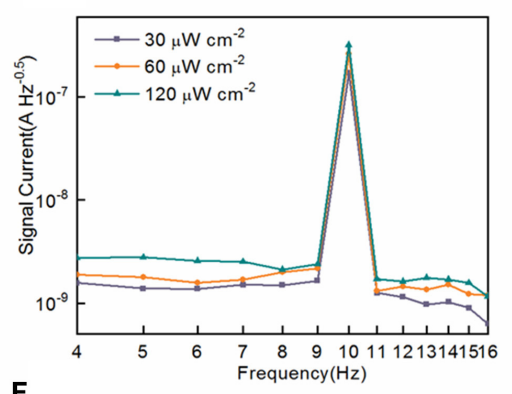

F

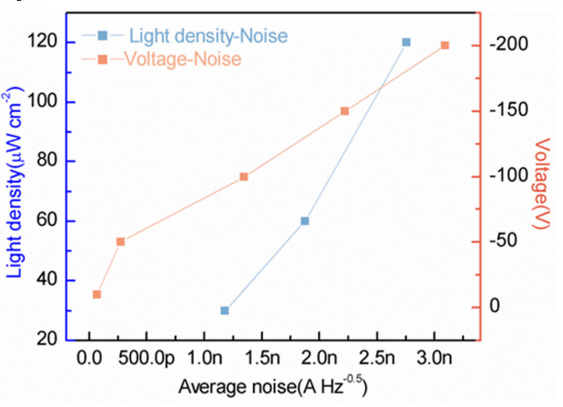

FIGURE 3 | (A) J-V curve and photocurrents at zero bias of the device under different illumination density. (B) Temporal response of the device under different illumination density at $460 \mathrm{~nm}$. (C) Signal current as a function of frequency under different illumination density at -100 V bias. (D) Temporal response of the device under different voltage bias at $460 \mathrm{~nm}$. (E) Signal current as a function of frequency under different voltage bias. (F) Plots of light density and voltage vs. average noise.

is fairly low of $6.97 \times 10^{-11} \mathrm{~A} \mathrm{~cm}^{-2}$ at $-0.5 \mathrm{~V}$ bias, which is one tenth of the $\mathrm{CH}_{3} \mathrm{NH}_{3} \mathrm{PbI}_{3}$ perovskite device reported before only with thick PCBM $(50 \mathrm{~nm}) / \mathrm{C} 60(130 \mathrm{~nm})$ layer as the ETL (Lin et al., 2015). What's more, we fabricate Device A with spincoated PCBM/C60 films on the $\mathrm{MAPbBr}_{2.5} \mathrm{Cl}_{0.5}$ substrate as the ETLS for comparison. The picture and J-V curve of Device A are shown in Figure S4. The dark current density of Device A at $-0.5 \mathrm{~V}$ bias is $4.24 \times 10^{-8} \mathrm{~A} \mathrm{~cm}^{-2}$, which is three orders of magnitude higher than our photodetector based on epitaxial mono-crystalline films. When the illumination density increases from $20 \mu \mathrm{Wcm}^{-2}$ to $120 \mu \mathrm{Wcm}^{-2}$, the photocurrent density increase from $1.81 \mu \mathrm{A} \mathrm{cm}{ }^{-2}$ to $5.12 \mu \mathrm{A} \mathrm{cm}^{-2}$ at $-50 \mathrm{~V}$ bias. Besides, the device could work at zero-bias as shown in the inset due to the in-built electrical field.

Figure 3B illustrates the temporal response of photodetectors based on epitaxial crystal layers with increased density of $460 \mathrm{~nm}$ illumination at $10 \mathrm{~Hz}$. In order to get the signal noise at low frequency of the device, the Fast Fourier Transform (FFT) method is used to transfer photocurrent density vs. time into signal current density vs. frequency (Dong et al., 2015b). And we analyze the signal current density ranges from 1 to $16 \mathrm{~Hz}$, as shown in Figure 3C. The noise current at low frequency increases approximately from 0.62 to $1.54 \mathrm{nA} \mathrm{Hz}^{-0.5}$ under current $30 \mu \mathrm{W}$ $\mathrm{cm}^{-2}$ illumination density. With light density increasing from 30 to $120 \mu \mathrm{W} \mathrm{cm}{ }^{-2}$, the noise current increases from 1.18 to $2.80 \mathrm{nA}$ $\mathrm{Hz}^{-0.5}$ at $-100 \mathrm{~V}$ bias.

Due to X-ray detection requires large voltage bias to collect charges induced in millimeter-thickness PSCs, here we characterize the noise current when the voltage bias varies from $-10 \mathrm{~V}$ to $-200 \mathrm{~V}$. Similar with Figures 3B,D shows the temporal response at $10 \mathrm{~Hz}$ with different voltages under 120 $\mu \mathrm{W} \mathrm{cm}^{-2}$ illumination density at $460 \mathrm{~nm}$. The average noise current increases from $68.7 \mathrm{pA} \mathrm{Hz}^{-0.5}$ to $3.24 \mathrm{nA} \mathrm{Hz}^{-0.5}$ in darkness and increases from $0.25 \mathrm{nA} \mathrm{Hz}^{-0.5}$ to $11.9 \mathrm{nA} \mathrm{Hz}^{-0.5}$ under illumination as shown in Figure 3E. In conclusion, the function of light density and voltage vs. average noise is shown in Figure 3F. This device with epitaxial mono-crystalline films is an excellent candidate for X-ray detection in terms of their low signal noise performance under high voltage bias (Kasap et al., 2011).

What's more, Responsivity (R), the ratio of photocurrent density and incident illumination density, indicates the efficiency of photodetectors responds to optical signal. And the formula is

$$
\mathbf{R}=\frac{\mathbf{J}_{\text {light }}-\mathbf{J}_{\text {dark }}}{\mathbf{P}}
$$

where $\mathbf{J}_{\text {light }}$ is photocurrent denisty, $\mathbf{J}_{\mathbf{d a r k}}$ is dark current denisty and $\mathbf{P}$ is incident illumination denisty (Dou et al., 2014). The area of photodetector is $25 \mathrm{~mm}^{2}$. As illustrated in Figure 3D, $\mathrm{R}$ can be calculated to approximately $87 \mathrm{~mA} / \mathrm{W}$ at $-100 \mathrm{~V}$ and $23 \mathrm{~mA} / \mathrm{W}$ at $-50 \mathrm{~V}$ bias with $120 \mu \mathrm{Wcm}^{-2}$ illumination at $460 \mathrm{~nm}$.

Figures 4A,B provide the time of flight (TOF) curves to measure the carrier mobility of semiconductor material. According to

$$
\mu=\frac{\mathbf{L}^{2}}{\mathbf{T}_{\mathrm{tof}} \mathbf{V}_{\mathrm{s}}}
$$



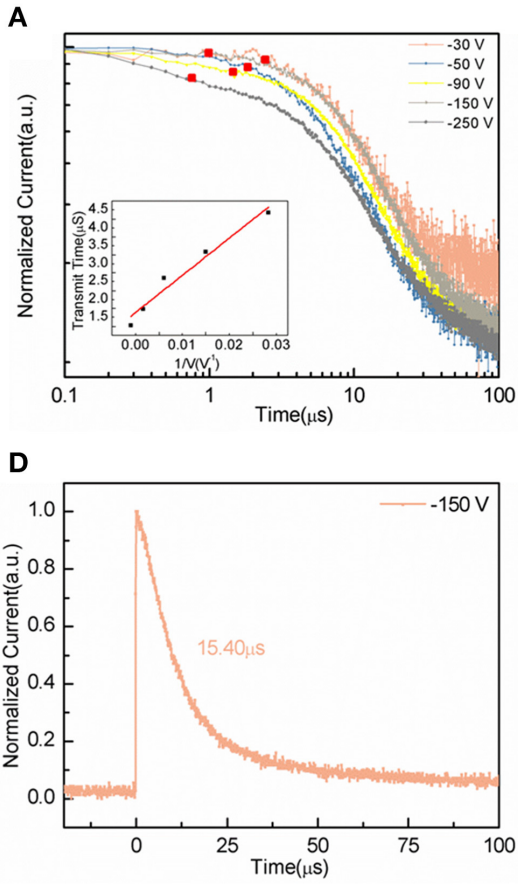

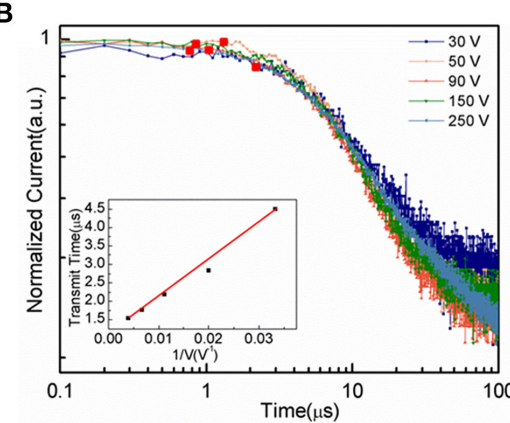

E

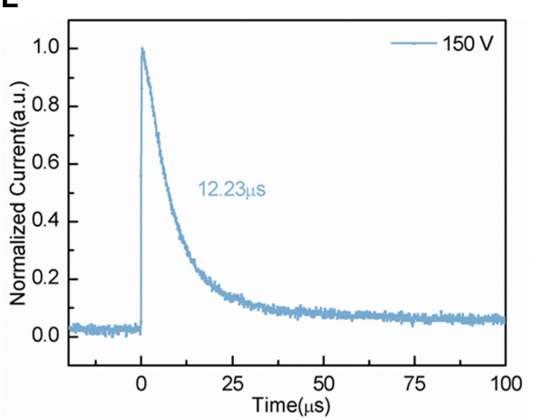

C

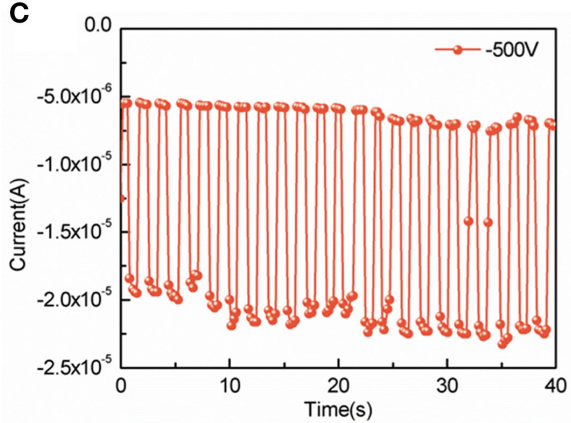

$\mathbf{F}$

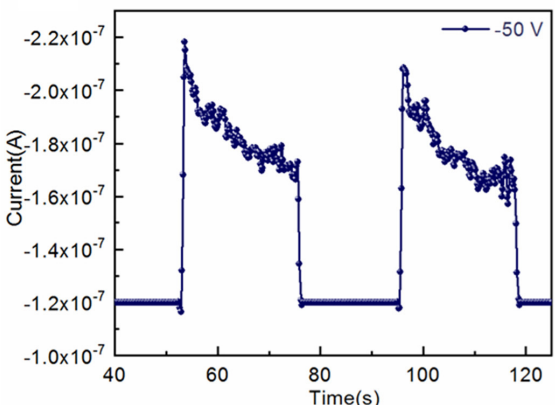

FIGURE 4 | (A) Electrons mobility caculated by time-of-flight (TOF) method. (B) Holes mobility caculated by TOF method. (C) Long-term transient response of the device at $-500 \mathrm{~V}$ bias. (D) Response time of the device at $-150 \mathrm{~V}$ bias. (E) Response time of the device at $150 \mathrm{~V}$ bias. (F) Transient response to $\mathrm{X}$-ray at $40 \mathrm{kV}$ and $1 \mathrm{~mA}$.

the carrier mobility $\mu$ can be directly calculated once we get the distance $\mathbf{L}$ and voltage bias $\mathbf{V}_{\mathbf{s}}$ between two electrodes and the carrier transit time $\mathbf{T}_{\text {tof. }}$ (Huang et al., 2015; Wei et al., 2016) As depicted in Figure 4A, the average mobility of electrons is calculated to $385 \mathrm{~cm}^{2} \mathrm{~V}^{-1} \mathrm{~s}^{-1}$ under reverse voltages. Similarly, Figure 4B shows the average holes mobility of $415 \mathrm{~cm}^{2} \mathrm{~V}^{-1} \mathrm{~s}^{-1}$ under forward voltages. The mobility here is larger than the those of devices based on PSCs results from the decreased dislocation between epitaxial grown films and lattice matched substrate (Dong et al., 2015a; Ji et al., 2018).

Figure 4C illustrates stable dark current and photocurrent of the photodetector under high work voltage of $-500 \mathrm{~V}$ bias at $460 \mathrm{~nm}$. The long-term stability of the device benefits from epitaxial mono-crystalline films replacement of function layers (Wang et al., 2019). As shown in Figures 4D,E, the response time of device is $15.40 \mu$ s under reverse bias and $12.23 \mu$ s under forward bias, which is 3 orders of magnitude faster than those of photodetectors based on perovskite polycrystalline thin films (Liu et al., 2019; Su et al., 2019).

The temporal X-ray response of our device is illustrated in Figure 4F. With $\mathrm{X}$-ray off, the dark current density is $480 \mathrm{nA} \mathrm{cm}^{-2}$ at $-50 \mathrm{~V}$ bias. And the photocurrent density reaches $880 \mathrm{nA} \mathrm{cm}^{-2}$ to X-ray at $40 \mathrm{kV}$ and $1 \mathrm{~mA}$ with dose rate of $6.7 \mu \mathrm{Gys}^{-1}$. The sensitivity of our device is $59.7 \mu \mathrm{C}$ $\mathrm{mGy}^{-1} \mathrm{~cm}^{-2}$ which is almost five times higher than X-ray detectors based on polycrystalline perovskite reported recently (Kim et al., 2017).

\section{CONCLUSION}

In summary, this work demonstrates a perovskite photodetector for X-ray detection fabricated with epitaxial monocrystalline films as electron-blocking layers. Due to lattice matching, we fabricates the device with epitaxial layers of high crystallinity and considerable thickness, which are characterized by XRD and SEM. Furthermore, our X-ray photodetector shows low dark current density of $6.97 \times$ $10^{-11} \mathrm{~A} \mathrm{~cm}^{-2}$ at $-0.5 \mathrm{~V}$ bias, high electrons mobility of $385.9 \mathrm{~cm}^{2} \mathrm{~V}^{-1} \mathrm{~s}^{-1}$, high responsivity of $870 \mathrm{~mA} / \mathrm{W}$ at $-100 \mathrm{~V}$ bias and remarkable X-ray sensitivity of $59.7 \mu \mathrm{C}$ $\mathrm{mGy}^{-1} \mathrm{~cm}^{-2}$ at $-50 \mathrm{~V}$ bias. This work offers a path of heteroepitaxial mono-crystalline films acting as electronblocking layers for solution-processed photodetectors based on OIHPSCs.

\section{DATA AVAILABILITY STATEMENT}

The raw data supporting the conclusions of this article will be made available by the authors, without undue reservation. 


\section{AUTHOR CONTRIBUTIONS}

YX, YL, and YP grew the perovskite single crystals. YX did the epitaxial experiments. YX and YP did the measurements. XW and YX analyzed these results. YX wrote this manuscript. All authors make comments on the manuscript.

\section{ACKNOWLEDGMENTS}

This work is supported by the National Key R\&D Program of China (Grant Nos. 2017YFC0111500 and 2016YFB0401600),

\section{REFERENCES}

Bao, C. X., Chen, Z. L., Fang, Y. J., Wei, H. T., Deng, Y. H., Xiao, X., et al. (2017). Low-noise and large-linear-dynamicrange photodetectors based on hybrid-perovskite thin-singlecrystals. Adv. Mater. 29:1703209. doi: 10.1002/adma.2017 03209

Dong, Q. F., Fang, Y. J., Shao, Y. C., Mulligan, P., Qiu, J., Cao, L., et al. (2015a). Electron-hole diffusion lengths $>175 \mathrm{mu} \mathrm{m}$ in solution-grown $\mathrm{CH} 3 \mathrm{NH} 3 \mathrm{PbI} 3$ single crystals. Science 347, 967-970. doi: 10.1126/science.aaa5760

Dong, R., Fang, Y., Chae, J., Dai, J., Xiao, Z., Dong, Q., et al. (2015b). High-gain and low-driving-voltage photodetectors based on organolead triiodide perovskites. Adv. Mater. 27, 1912-1918. doi: 10.1002/adma.201405116

Dou, L. T., Yang, Y., You, J. B., Hong, Z. R., Chang, W. H., Li, G., et al. (2014). Solution-processed hybrid perovskite photodetectors with high detectivity. Nat. Commun. 5:404. doi: 10.1038/ncomms6404

Fang, Y. J., Dong, Q. F., Shao, Y. C., Yuan, Y. B., and Huang, J. S. (2015). Highly narrowband perovskite single-crystal photodetectors enabled by surface-charge recombination. Nat. Photonics 9:679. doi: 10.1038/nphoton. 2015.156

Hu, W., Qin, X., Yao, Y., and Dong, H. (2016). Perovskite Photodetectors based on CH3NH3PbI3 single crystals. Chem. An Asian J 11:2675-2679.

Huang, J., Shao, Y., and Dong, Q. (2015). Organometal trihalide perovskite single crystals: a next wave of materials for $25 \%$ efficiency photovoltaics and applications beyond? J. Phys. Chem. Lett. 6:3218-3227. doi: 10.1021/acs.jpclett. 5 b01419

Jariwalad, M., and Hersammc. (2017). Mixed-dimensional van der Waals heterostructures. Nat. Mater. 16, 170-181. doi: 10.1038/nmat4703

Ji, L., Hsu, H. Y., Lee, J. C., Bard, A. J., and Yu, E. T. (2018). Highperformance photodetectors based on solution-processed epitaxial grown hybrid halide perovskites. Nano Lett. 18, 994-1000. doi: 10.1021/acs.nanolett. $7 \mathrm{~b} 04445$

Jiang, S., Wang, X., Wu, Y., Li, Y., Zhang, Q., Li, G., et al. (2019). Balance lead in solution-processed $\mathrm{CH} 3 \mathrm{NH} 3 \mathrm{PbBrxCl}(3-\mathrm{x})$ single crystals for high performance X-ray detection. Mater. Lett 236:26-29. doi: 10.1016/j.matlet.2018.10.055

Kasap, S., Frey, J. B., Belev, G., Tousignant, O., Mani, H., Greenspan, J., et al. (2011). Amorphous and polycrystalline photoconductors for direct conversion flat panel x-ray image sensors. Sensors (Basel) 11, 5112-5157. doi: 10.3390/s110505112

Kim, Y. C., Kim, K. H., Son, D. Y., Jeong, D. N., Seo, J. Y., Choi, Y. S., et al. (2017). Printable organometallic perovskite enables large-area, low-dose X-ray imaging. Nature 550, 87. doi: 10.1038/nature24032

Li, Y.-W., Wang, X., Li, G.-W., Wu, Y., Pan, Y.-Z., Xu, Y.-B., et al. (2020). Fast liquid phase epitaxial growth for perovskite single crystals. Chinese Phys. Lett. 37:018101. doi: 10.1088/0256-307X/37/1/018101

Lin, Q., Armin, A., Lyons, D. M., Burn, P. L., and Meredith, P. (2015). Low noise, IR-blind organohalide perovskite photodiodes for visible light detection and imaging. Adv. Mater. Weinheim 27, 2060-2064. doi: 10.1002/adma.2014 05171

Liu, Y., Zhang, Y., Yang, Z., Feng, J., Xu, Z., Li, Q., et al. (2019). Lowtemperature-gradient crystallization for multi-inch high-quality perovskite the National Natural Science Foundation Project (Grant Nos. 61775034, 61571124, and 61674029), International Cooperation Program of Jiangsu Province (Grant No. BZ2018056), and the NSFC Research Fund for International Young Scientists (Grant No. 61750110537).

\section{SUPPLEMENTARY MATERIAL}

The Supplementary Material for this article can be found online at: https://www.frontiersin.org/articles/10.3389/fchem. 2020.00811/full\#supplementary-material single crystals for record performance photodetectors. Mater. Today 22, 67-75. doi: 10.1016/j.mattod.2018.04.002

Maculan, G., Sheikh, A. D., Abdelhady, A. L., Saidaminov, M. I., Hague, M. A. Murali, B., et al. (2015). CH3NH3PbCl3 single crystals: inverse temperature crystallization and visible-blind UV-photodetector. J. Phys. Chem. Lett. 6 , 3781-3786. doi: 10.1021/acs.jpclett.5b01666

Miao, J. L., and Zhang, F. J. (2019). Recent progress on highly sensitive perovskite photodetectors. J. Mater. Chem. C 7, 1741-1791. doi: 10.1039/C8TC0 6089D

Nakamura, K., Toda, S., and Yamanouchi, M. (2001). A two-dimensional optical fibre microphone array with matrix-style data readout. Meas. Sci. Technol. 12, 859-864. doi: 10.1088/0957-0233/12/7/319

Pan, D., Fu, Y., Chen, J., Czech, K. J., Wright, J. C., and Jin, S. (2018). Visualization and studies of ion-diffusion kinetics in cesium lead bromide perovskite nanowires. Nano Lett. 18, 1807-1813. doi: 10.1021/acs.nanolett. $7 \mathrm{~b} 05023$

Pellegrino, G., Colella, S., Deretzis, I., Condorelli, G. G., Smecca, E., Gigli, G., et al. (2015). Texture of MAPbI3 Layers assisted by Chloride on Flat $\mathrm{TiO}_{2}$ Substrates. J. Phys. Chem. C 119:19808-19816.

Shewmon, N. T., Yu, H., Constantinou, I., Klump, E., and So, F. (2016). Formation of perovskite heterostructures by ion exchange. ACS Appl. Mater. Interfaces 8, 33273-33279. doi: 10.1021/acsami.6b10034

Stoumpos, C. C., Malliakas, C. D., Peters, J. A., Liu, Z. F., Sebastian, M., Im, J., et al. (2013). Crystal growth of the perovskite semiconductor CsPbBr3: a new material for high-energy radiation detection. Crystal Growth Design 13, 2722-2727. doi: $10.1021 / \mathrm{cg} 400645 \mathrm{t}$

Su, J., Bai, Y., Huang, Y., Wang, D., Kuang, W., and Xu, L. (2019). Morphology, optical and photoelectric properties of $\mathrm{CH} 3 \mathrm{NH} 3 \mathrm{PbBr} 3$ single crystal. Physica B 571, 307-311. doi: 10.1016/j.physb.2019. 04.014

Sutherland, B. R., and Sargent, E. H. (2016). Perovskite photonic sources. Nat. Photonics 10, 295-302. doi: 10.1038/nphoton.2016.62

Wang, H., and Kim, D. H. (2017). Perovskite-based photodetectors: materials and devices. Chem. Soc. Rev. 46, 5204-5236. doi: 10.1039/C6CS0 0896H

Wang, Q., Shao, Y., Xie, H., Lyu, L., Liu, X., Gao, Y., et al. (2014). Qualifying composition dependent $\mathrm{p}$ and $\mathrm{n}$ self-doping in $\mathrm{CH} 3 \mathrm{NH} 3 \mathrm{PbI} 3$. Appl. Phys. Lett. 105:163508. doi: 10.1063/1.4899051

Wang, X., Li, Y., Xu, Y., Pan, Y., Wu, Y., Li, G., et al. (2019). Organometallic perovskite single crystals grown on lattice-matched substrate for photodetection. Nano Mater. Sci. doi: 10.1016/j.nanoms.2019. 10.007

Wang, X., Zhao, D. W., Qiu, Y. P., Huang, Y., Wu, Y., Li, G. W., et al. (2018). PIN diodes array made of perovskite single crystal for X-Ray imaging. Phys. Status Solidi Rapid Res. Lett. 12:1800380. doi: 10.1002/pssr.201800380

Wei, H. T., Fang, Y. J., Mulligan, P., Chuirazzi, W., Fang, H. H., Wang, C. C., et al. (2016). Sensitive X-ray detectors made of methylammonium lead tribromide perovskite single crystals. Nat. Photonics 10:333. doi: 10.1038/nphoton. 2016.41

Wei, W., Zhang, Y., Xu, Q., Wei, H., Fang, Y., Wang, Q., et al. (2017). Monolithic integration of hybrid perovskite single crystals with heterogenous 
substrater for highly sensitive X-ray imaging. Nat. Photonics 11, 315-321. doi: 10.1038/nphoton.2017.43

Xin, W., Wu, Y., Li, G., Wu, J., and Zhang, X. (2018). Ultrafast Ionizing Radiation Detection by $p-n$ Junctions Made with Single Crystals of SolutionProcessed Perovskite.

Zhang, Y., Lu, D., Gao, M., Lai, M., Lin, J., Lei, T., et al. (2019). Quantitative imaging of anion exchange kinetics in halide perovskites. Proc. Natl. Acad. Sci. U.S.A. 116, 12648-12653. doi: 10.1073/pnas.1903448116

Zhao, D., Sexton, M., Park, H. U., Baure, G., and So, F. (2014). High-efficiency solution-processed planar perovskite solar cells with a polymer hole transport layer. Adv. Energy Mater 5:1401855. doi: 10.1002/aenm.201401855
Conflict of Interest: The authors declare that the research was conducted in the absence of any commercial or financial relationships that could be construed as a potential conflict of interest.

Copyright $\odot 2020 \mathrm{Xu}$, Wang, Pan, Li, Emeka Elemike, Li, Zhang, Chen, Zhao and Lei. This is an open-access article distributed under the terms of the Creative Commons Attribution License (CC BY). The use, distribution or reproduction in other forums is permitted, provided the original author(s) and the copyright owner(s) are credited and that the original publication in this journal is cited, in accordance with accepted academic practice. No use, distribution or reproduction is permitted which does not comply with these terms. 